\title{
Effet de la concentration en phospholipides de babeurre dans le lait de fromagerie sur la production et la composition de fromages allégés de type Cheddar
}

\author{
Sophie TurCota, Sylvie L. TurgeON ${ }^{\mathrm{b}}$, Daniel St-Gelais ${ }^{\mathrm{a}^{*}}$ \\ ${ }^{a}$ Agriculture et Agroalimentaire Canada, Centre de Recherche et de Développement \\ sur les Aliments, 3600 bld Casavant Ouest, Saint-Hyacinthe, Qc, Canada J2S 8E3 \\ $\mathrm{b}$ Centre de Recherche en Sciences et Technologie du Lait, Pavillon Paul-Comtois, \\ Université Laval, Sainte-Foy, Qc, Canada G1K 7P4
}

(Reçu le 28 janvier 2000 ; accepté le 8 septembre 2000)

\begin{abstract}
Effect of buttermilk phospholipid concentrations in cheese milk on production and composition of low fat Cheddar cheese. Skim milk, sweet buttermilk, UF milk retentate and UF buttermilk retentate were used to produce four cheese milks, in which proteins and fat were standardised, respectively to 40 and $20 \mathrm{~g} \cdot \mathrm{kg}^{-1}$. However, the concentration of phospholipids in these cheese milks was adjusted to $0.3 ; 0.8 ; 1.0$ and $1.2 \mathrm{~g} \cdot \mathrm{kg}^{-1}$. These different cheese milks were used to produce reduced fat Cheddar cheeses with $50 \%$ less fat. Results of compositional analyses indicated that the use of buttermilk affected low fat cheese composition. An average of $44 \%$ of phospholipids were retained in the cheese curds, whatever the type of cheese milk used to produce cheeses. The moisture content increased from 420 to $520 \mathrm{~g} \cdot \mathrm{kg}^{-1}$ with concentration of phospholipids, in spite of cheese manufacturing modifications (different coagulation time and whey off $\mathrm{pH}$ ) carried out. However, the fat lost in whey was higher when cheese were made from milks rich in phospholipids. Low fat cheese yields increased from 9.4 to $11.7 \mathrm{~kg} \cdot 100 \mathrm{~kg}^{-1}$ with concentration of phospholipids, but this increase was due to high water content in cheese, but not to better retention of the other milk components. These results indicate that buttermilk is an excellent source of phospholipids, which allows to increase low fat cheese moisture.
\end{abstract}

\section{reduced fat cheese / buttermilk / phospholipid / ultrafiltration}

Résumé — Du lait écrémé, du babeurre et des rétentats de lait UF et de babeurre UF ont été combinés pour produire quatre laits de fromagerie tous standardisés en protéines à $40 \mathrm{~g} \cdot \mathrm{kg}^{-1}$ et en matières grasses à $20 \mathrm{~g} \cdot \mathrm{kg}^{-1}$ mais dont les concentrations en phospholipides ont été ajustées à 0,$3 ; 0,8 ; 1,0$ et $1,2 \mathrm{~g} \cdot \mathrm{kg}^{-1}$. Ces différents laits de fromagerie ont été utilisés pour fabriquer des fromages allégés de

\footnotetext{
* Correspondance et tirés-à-part
}

Tél. : 450 773-1105 ; fax : 450 773-8461 ; e-mail : stgelaisd@em.agr.ca 
type Cheddar dont la teneur en matières grasses a été réduite de $50 \%$. L'ajout de phospholipides sous forme de babeurre a modifié la composition des fromages allégés. En moyenne, $44 \%$ des phospholipides ont été retenus dans tous les caillés, quel que soit le lait de fromagerie utilisé pour la production fromagère. La teneur en humidité des fromages est passée de 420 à $520 \mathrm{~g} \cdot \mathrm{kg}^{-1}$ avec l'augmentation de la teneur en phospholipides, malgré les modifications apportées lors de certaines étapes de la fabrication fromagère (temps de coagulation et $\mathrm{pH}$ de soutirage différents). Par contre, plus la teneur en phospholipides était élevée dans le lait de fromagerie, plus les pertes en matières grasses étaient également élevées dans le lactosérum. Les rendements fromagers bruts sont donc passés de 9,4 à $11,7 \mathrm{~kg} \cdot 100 \mathrm{~kg}^{-1}$ avec l'augmentation de la teneur en phospholipides, mais cette augmentation n'était due qu'à la teneur élevée en eau des fromages et non pas à une meilleure rétention des autres constituants du lait. Les résultats confirment donc que le babeurre est une excellente source de phospholipides permettant d'augmenter facilement la teneur en humidité d'un fromage allégé de type Cheddar.

\section{fromage allégé / babeurre / phospholipide / ultrafiltration}

\section{INTRODUCTION}

Durant les 15 à 20 dernières années, la demande pour des fromages dont la teneur en matières grasses est réduite n'a cessé d'augmenter [15]. Cependant, la réduction en matières grasses du lait de fromagerie entraîne des défauts de texture et de saveur du fromage de type Cheddar. En effet, les fromages à pâte ferme à faible teneur en matières grasses ont une matrice protéique très compacte qui leur confère une texture très ferme et une sensation en bouche plutôt sèche [10,29]. Par conséquent, il a été suggéré d'augmenter la teneur en humidité des fromages dits allégés pour améliorer leur qualité organoleptique [9, 29]. La teneur en eau des fromages allégés et même standards peut être augmentée de 1 à $6 \%$ en ajoutant dans du lait de fromagerie des protéines sériques préalablement dénaturées provenant du lait, de lactosérums ou de concentrés protéiques de lactosérum $[1,7$, $8,17,26]$. L'ajout de phospholipides (PPL) d'origine végétale (lécithine de soja) dans le lait de fromagerie permettrait également d'augmenter la teneur en humidité de fromages allégés et ainsi d'améliorer leur texture. Toutefois, l'ajout de lécithine de soja serait à l'origine de défauts de saveur du fromage [16]. Or, il existe une autre source de phospholipides d'origine laitière qui est le babeurre.

Le babeurre est un sous-produit de la fabrication du beurre qui contient la majeure partie des phospholipides issus de la membrane des globules gras du lait [11]. Les phospholipides sont des molécules possédant une extrémité hydrophile (portant des groupements polaires) et une autre hydrophobe (par la présence de chaînes aliphatiques). Ces molécules sont des agents interfaciaux stabilisant les émulsions. Dans le lait, ils composent avec des protéines une membrane émulsifiant les glycérides [12, 14]. De plus, les phospholipides possèdent une importante capacité à retenir l'eau, ce qui permettrait d'augmenter la teneur en humidité des fromages allégés $[16,18]$.

Mistry et al. [27] ont étudié l'effet du taux de substitution du lait par du babeurre UF sur la production du fromage de type Cheddar à teneur réduite en matières grasses. Ils ont obtenu les meilleurs résultats lorsque la substitution était comprise entre 3 et $5 \%$. Cependant, par rapport au lait témoin, l'ajout de babeurre UF a fait augmenter la teneur en protéines mais diminuer la teneur en matières grasses des laits de fromagerie. Le rapport protéines/matières grasses pour chaque lait de fromagerie n'était donc pas constant. 
Le but de la présente étude était de déterminer l'effet de l'ajout de babeurre comme source de phospholipides sur la production, la composition et les rendements fromagers de fromage allégé de type Cheddar tout en maintenant constantes, dans les laits de fromagerie, les concentrations en protéines et en matières grasses (rapport protéines/matière grasse constant).

\section{MATÉRIEL ET MÉTHODES}

\subsection{Ingrédients laitiers et laits de fromagerie}

Pour chaque journée de fabrication, quatre laits de fromagerie ont été préparés à partir de crème fraîche, de lait écrémé et de babeurre provenant de la laiterie Mont St-Hilaire (St-Hyacinthe, Qc, Canada) et de rétentats de lait UF et de babeurre UF. Ces laits ont été préparés dans le but d'ajuster la teneur en phospholipides de 0,3 à $1,2 \mathrm{~g} \cdot \mathrm{kg}^{-1}$ tout en maintenant constantes leurs teneurs en protéines et en matières grasses respectivement à 40 et $20 \mathrm{~g} \cdot \mathrm{kg}^{-1}$. Ces différents laits ont été utilisés pour produire des fromages de type Cheddar allégés en matières grasses de $50 \%$.

Les rétentats de lait UF (LUF) et de babeurre UF (BUF) ont été obtenus, en concentrant par un facteur volumique de 5 (FCV 5X) à l'aide d'une unité pilote d'ultrafiltration (Alfa-Laval, Uppsala, Suède, PM 50, 2,46 $\mathrm{m}^{2}$ ), respectivement $100 \mathrm{~L}$ de lait écrémé et $100 \mathrm{~L}$ de babeurre provenant de la laiterie Mont St-Hilaire (St-Hyacinthe). L'ultrafiltration a été réalisée à $50^{\circ} \mathrm{C}$. Le perméat a été pesé afin d'évaluer le facteur de concentration du lait. Les rétentats LUF et BUF ont été congelés et entreposés à $-40{ }^{\circ} \mathrm{C}$ jusqu' au moment de leur utilisation.

Le lait de fromagerie LLUF, contenant $0,3 \mathrm{~g} \cdot \mathrm{kg}^{-1}$ de PPL, a été obtenu en mélangeant du lait écrémé et du rétentat LUF. Le lait LBUF, contenant $0,8 \mathrm{~g} \cdot \mathrm{kg}^{-1}$, a été obtenu en mélangeant du lait écrémé et du rétentat BUF. Le lait LB50, contenant $1,0 \mathrm{~g} \cdot \mathrm{kg}^{-1}$, provenait d'un mélange de lait écrémé et de babeurre liquide dans une proportion de 50:50 auquel a été ajouté un mélange de rétentat de lait UF et de rétentat de babeurre UF également dans une proportion de 50:50. Enfin, le lait BLUF, contenant $1,2 \mathrm{~g} \cdot \mathrm{kg}^{-1}$, a été préparé en mélangeant du babeurre liquide avec du rétentat LUF.

Lors de la préparation des laits de fromagerie, une solution de chlorure de calcium à $33 \%$ m/m (Rhône-Poulenc, Marshall Products, Madison, WI), a été ajoutée aux laits LB50 et BLUF afin d'ajuster leur teneur en calcium à $1,4 \mathrm{~g} \cdot \mathrm{kg}^{-1}$ comme pour les laits LLUF et LBUF. Les laits de fromagerie ont ensuite été conservés $18 \mathrm{~h}$ à $4{ }^{\circ} \mathrm{C}$ jusqu' au moment de leur utilisation.

\subsection{Fabrication fromagère}

Le jour précédant les fabrications, $2,5 \mathrm{~kg}$ de lait écrémé reconstitué $\left(120 \mathrm{~g} \cdot \mathrm{kg}^{-1} \mathrm{ES}\right)$ préalablement stérilisé à l'autoclave $\left(10 \mathrm{~min}, 112^{\circ} \mathrm{C}\right)$ a été inoculé à un taux de $3,0 \mathrm{~g} \cdot \mathrm{kg}^{-1}$ avec un ferment lactique congelé Redi-set 120 (Chr. Hansen's Laboratory, ON, Canada). Le lait inoculé a été incubé à $21^{\circ} \mathrm{C}$ pendant $14 \mathrm{~h} 30 \mathrm{~min}$, puis réfrigéré et maintenu à $4{ }^{\circ} \mathrm{C}$ jusqu'au moment de son utilisation. Le $\mathrm{pH}$ du ferment était à 4,85 $\pm 0,5$.

Le jour des fabrications, le $\mathrm{pH}$ des laits de fromagerie a été ajusté à 6,60 avec une solution $(20 \% \mathrm{~m} / \mathrm{m})$ d'hydroxyde de calcium (Penflow 51, Atochem Canada Ltée, Qc, Canada). Les fabrications fromagères ont été réalisées dans des cuves de $20 \mathrm{~kg}$ contenant chacune $18 \mathrm{~kg}$ de lait. Les laits ont ensuite été traités thermiquement à $65^{\circ} \mathrm{C}$ pendant $30 \mathrm{~min}$ (pasteurisation en cuve) puis refroidis et maintenu à $32^{\circ} \mathrm{C}$. Les laits pasteurisés ont été ensemencés à un taux de $27,8 \mathrm{~g} \cdot \mathrm{kg}^{-1}$ avec le ferment préparé la veille. Après 30 min de maturation, 
du chlorure de calcium et de la présure (Chymostar Classic ; 250 IMCU/mL; Rhône-Poulenc, Marshall Products, Madison, Inc.), ont été ajoutés aux laits à un taux respectif de 0,26 et $0,20 \mathrm{~mL} \cdot \mathrm{kg}^{-1}$ de lait.

Des essais préliminaires ont démontré qu'en utilisant un procédé de fabrication conventionnel [22], certains fromages allégés en matières grasses avaient des teneurs en humidité trop élevées, jusqu'à $600 \mathrm{~g} \cdot \mathrm{kg}^{-1}$. Des paramètres de fabrication ont donc été modifiés afin d'abaisser la teneur en humidité de ces fromages. Ainsi, les caillés LLUF, LBUF, LB50 et BLUF ont été coupés en cubes de $1 \mathrm{~cm}^{3}$ respectivement après 41, 33, 36 et 29 min de coagulation. Dix minutes après le découpage, la température de toutes les cuves a été progressivement élevée à $38^{\circ} \mathrm{C}$ pendant 30 min. Après respectivement, 73, 107, 123 et 154 min en moyenne, le lactosérum pour les caillés LLUF, LBUF, LB50 et BLUF a été soutiré à des valeurs de $\mathrm{pH}$ respectif de 6,$1 ; 6,0 ; 5,9$ et 5,8 . Les caillés LLUF, LBUF, LB50 et BLUF ont ensuite été empilés et cheddarisés, respectivement pendant 133, 110, 92 et 91 min pour atteindre une valeur de $\mathrm{pH}$ identique de 5,4. Tous les caillés ont été hachés et salés à un taux de $28 \mathrm{~g} \cdot \mathrm{kg}^{-1}$ pour le caillé LLUF (moins humide que les autres caillés) et $30 \mathrm{~g} \cdot \mathrm{kg}^{-1}$ pour les trois autres caillés (LBUF, LB50 et BLUF). Dix minutes après le salage, les caillés ont été placés dans des moules et pressés à $206 \mathrm{KPa}$ pendant $16 \mathrm{~h}$. La durée moyenne de fabrication pour tous les fromages allégés a été de $285 \mathrm{~min}$. Les fromages ont ensuite été scellés sous vide et entreposés à $4{ }^{\circ} \mathrm{C}$ pendant 12 semaines.

\subsection{Analyses de composition}

Les teneurs en protéines et en matières grasses des ingrédients laitiers frais (lait écrémé, babeurre et crème) utilisés pour la formulation des différents laits de fromagerie à chaque jour des fabrications, ont été déterminées en triple à l'aide d'un analy- seur infrarouge (Dairylab 2, Multispec Ltée, Wheldrake, York, England).

La composition des concentrés UF (LUF et BUF), des laits de fromagerie, des lactosérums et des fromages a été déterminée en double. L'azote total (NT) ainsi que l'azote non caséique (NNC) obtenu après précipitation des caséines à $\mathrm{pH}$ 4,6 ont été dosés par la méthode macroKjeldahl [4]. La teneur en azote caséique (NC) a été calculée par différence [NC = (NT - NNC)]. Les différentes teneurs en azote ont été exprimées en protéines en utilisant le facteur de conversion de 6,38. Les cendres ont été déterminées après incinération dans un four à haute température $\left(550^{\circ} \mathrm{C}\right)$ pendant $16 \mathrm{~h}$. Les extraits secs (ES) ont été déterminés après séchage au four à $110^{\circ} \mathrm{C}$ pendant $5 \mathrm{~h}$ pour les liquides et $18 \mathrm{~h}$ pour les fromages. La teneur en matières grasses (MG) a été obtenue par la méthode Mojonnier [5]. La teneur en lactose des ingrédients et des laits de fromagerie a été calculée par différence [Lactose $=(E S-$ $\mathrm{MG}-(\mathrm{NT} \times 6,38)$ - cendres $)]$.

Les minéraux (Ca, $\mathrm{P}, \mathrm{Mg}$ et $\mathrm{K})$ dans les ingrédients laitiers et les fromages ont été analysés à l'aide d'un appareil de chromatographie en phase liquide de type ionique (Dionex DX 500, Sunnyvale, CA) selon un protocole développé dans nos laboratoires. Pour les fromages, les minéraux ont été dosés directement à partir des cendres. Les échantillons ( $2 \mathrm{~g})$ de fromage ont d'abord été incinérés dans un four à haute température $\left(550^{\circ} \mathrm{C}\right)$ pendant $16 \mathrm{~h}$. Par la suite, les cendres ont été resuspendues dans $5 \mathrm{~mL}$ de TCA $20 \%$. Les solutions ont été transférées dans une fiole jaugée de $100 \mathrm{~mL}$ et complétée à $100 \mathrm{~mL}$ avec de l'eau déionisée. Les échantillons ont alors été dilués pour que leur teneur en minéraux se situe dans la courbe étalon. Un volume de $5 \mathrm{~mL}$ de chaque échantillon a été filtré sur filtre de $0.45 \mu \mathrm{m}$ dans des Polyvials avec bouchons (Dionex, P/N 043190) et injecté dans l'appareil à l'aide de l'injecteur automatique AS40 (Dionex DX 500, Sunnyvale, CA). 
Les anions ont d'abord été séparés sur colonne anionique AS4A-SC (Dionex, P/N 043174) couplée à un suppresseur anionique ASRS-1 (Dionex, P/N 043189) puis ensuite détectés et quantifiés par un détecteur de conductivité CD20 (Dionex DX 500, Sunnyvale, CA). Les cations ont été séparés sur colonne cationique CS12A (Dionex, P/N 046073) couplée à un suppresseur cationique CSRS-1 (Dionex, P/N 043190) puis ensuite détectés et quantifiés par le même détecteur de conductivité que les anions. La phase analytique des anions était constituée de $\mathrm{Na}_{2} \mathrm{CO}_{3} 1,8 \mathrm{mN}$ et de $\mathrm{NaOH} 0,5 \mathrm{mN}$ tandis que la phase analytique des cations était constituée de $\mathrm{H}_{2} \mathrm{SO}_{4}$ $22 \mathrm{mN}$. La courbe étalon pour les ions phosphate $\left(\mathrm{PO}_{4}^{-}\right)$se situait de 20 à 100 ppm. Les concentrations en phosphates ont été converties en phosphore. La courbe étalon pour les cations se situait de 5 à 100 ppm pour le sodium $\left(\mathrm{Na}^{+}\right)$, le calcium $\left(\mathrm{Ca}^{+}\right)$et le potassium $\left(\mathrm{K}^{+}\right)$tandis que celle du magnésium $\left(\mathrm{Mg}^{+}\right)$variait de 1 à $20 \mathrm{ppm}$.

La concentration de sel dans les fromages a été déterminée par un chlorure mètre analyseur de sel Corning (Nelson-Jameson, Inc. Marshfield, WI). Le pH des fromages a été mesuré à l'aide d'un pH-mètre (modèle pH C2431, Radiometer, Copenhagen, Danemark).

Les phospholipides contenus dans les ingrédients (lait écrémé, babeurre, crème et rétentats UF), les laits de fromagerie et les lactosérums de soutirage et de pressage ont été dosés sur des échantillons lyophilisés à l'aide d'un lyophilisateur Virtis 12 (The Virtis Company, Gardier, NY). Les lipides totaux contenus dans les échantillons lyophilisés ont d'abord été extraits sur colonne de Célite 545 à l'aide d'un mélange méthanol/chloroforme $(2: 1 \mathrm{v} / \mathrm{v})$ selon la méthode décrite par Pierre et al. [28]. Le phosphore contenu dans l'extrait lipidique a été dosé par colorimétrie selon la méthode de Ames [3]. La quantité de phosphore obtenue a été multipliée par un facteur de conversion de 25 pour déterminer la teneur en phospholi- pides par rapport aux lipides totaux des échantillons [34]. La teneur en PPL dans les fromages a été estimée par différence [teneur en PPL des fromages $=($ teneur en PPL du lait - teneur en PPL des lactosérums de soutirage et de pressage)].

\subsection{Rendements fromagers}

Le rendement fromager brut $\left(\mathrm{RD}_{\mathrm{B}}\right)$ est exprimé en $\mathrm{kg}$ de fromage après pressage par $100 \mathrm{~kg}$ de lait. Le rendement fromager ajusté $\left(\mathrm{RD}_{\mathrm{A}}\right)$ a été calculé à l'aide de la formule suivante [27] en tenant compte des teneurs en humidité et en sel désirées, soit 470 et $22,5 \mathrm{~g} \cdot \mathrm{kg}^{-1}$ :

$$
\mathrm{RD}_{\mathrm{A}}=\mathrm{RD}_{\mathrm{B}} \times \frac{\left(1000-\mathrm{H}_{\mathrm{act}}-\mathrm{S}_{\mathrm{act}}\right)}{(1000-470-22,5)}
$$

d'où $\mathrm{H}_{\text {act }}$ et $\mathrm{S}_{\text {act }}$ sont respectivement la teneur en humidité $\left(\mathrm{g} \cdot \mathrm{kg}^{-1}\right)$ et en sel $\left(\mathrm{g} \cdot \mathrm{kg}^{-1}\right)$ mesurée dans les fromages.

Les taux de récupération des protéines $\mathrm{K}_{\mathrm{P}}$ et de la matière grasses $\mathrm{K}_{\mathrm{G}}$ dans les fromages ont été calculés selon les formules suivantes [33] :

$$
\begin{gathered}
K_{P}=\frac{\left(P_{F} \times R D_{B}\right)}{P_{L}} \\
K_{G}=\frac{\left(G_{F} \times R D_{B}\right)}{G_{L}}
\end{gathered}
$$

d'où $P_{F}$ et $G_{F}$ représentent respectivement la teneur en protéines $\left(\mathrm{g} \cdot \mathrm{kg}^{-1}\right)$ et en matières grasses $\left(\mathrm{g} \cdot \mathrm{kg}^{-1}\right)$ dans le fromage tandis que $P_{L}$ et $G_{L}$ représentent la concentration en protéines $\left(\mathrm{g} \cdot \mathrm{kg}^{-1}\right)$ et en matières grasses $\left(\mathrm{g} \cdot \mathrm{kg}^{-1}\right)$ dans le lait de fromagerie.

\subsection{Analyses statistiques}

Un dispositif complètement aléatoire, répété 4 fois, a été utilisé pour évaluer l'effet de la teneur en phospholipides du lait de fromagerie sur la composition des fromages allégés et des lactosérums ainsi que sur 
les rendements fromagers. Au total, 16 fabrications ont été réalisées. À l'intérieur d'une journée (répétition), les quatre types de lait ont été évalués. Les analyses statistiques ont été effectuées en utilisant la procédure GLM à l'aide du logiciel SAS [32].

\section{RÉSULTATS}

\subsection{Composition des ingrédients et des laits de fromagerie}

La composition de tous les ingrédients utilisés pour la préparation des laits de fromagerie est présentée dans le tableau I. Le babeurre a une composition voisine de celle du lait écrémé. Cependant, il contient une quantité plus importante de phospholipides et de matières grasses mais moins de calcium et de protéines. Ces résultats sont comparables à ceux obtenus par d'autres chercheurs [2, 13]. Le tableau I montre également que le babeurre aurait été concentré 6 fois par UF au lieu de 5 fois. Cela explique pourquoi, la teneur en protéines du rétentat BUF était plus élevée que celle du rétentat LUF. La crème utilisée pour cette étude avait en moyenne une teneur en MG de $390,7 \pm 11,2 \mathrm{~g} \cdot \mathrm{kg}^{-1}$ et globalement sa composition correspondait à celle obtenue dans la littérature [33].

Tous ces ingrédients ayant des teneurs en protéines, en matières grasses et en phospholipides différentes ont pu être utilisés pour produire différents laits de fromagerie (Tab. II). Tous les laits de fromagerie avaient des teneurs en protéines totales et en matières grasses proches des valeurs cibles, soit de $40 \mathrm{~g} \cdot \mathrm{kg}^{-1}$ pour les protéines totales et de $20 \mathrm{~g} \cdot \mathrm{kg}^{-1}$ pour les matières grasses, à l'exception du lait BLUF dont les concentrations en protéines totales et en matières grasses étaient légèrement plus faibles. Par contre tous les laits de fromagerie avaient une teneur en caséines semblable.

La différence dans la teneur en protéines totales des laits proviendrait de l'azote non caséique qui était également plus faible pour le lait BLUF. De plus, les teneurs en lactose et en calcium étaient significative-

Tableau I. Composition $\left(\mathrm{g} \cdot \mathrm{g}^{-1}\right)$ des ingrédients utilisés pour la préparation des laits de fromagerie.

Table I. Composition $\left(\mathrm{g} \cdot \mathrm{kg}^{-1}\right)$ of ingredients used to make cheese milks.

\begin{tabular}{lccccc}
\hline Constituant & Lait écrémé* $^{*}$ & Babeurre $^{*}$ & LUF $^{1}$ & BUF $^{1}$ & Crème $^{*}$ \\
\hline Protéines totales & 32,3 & 26,8 & 169,2 & 173,5 & 18,6 \\
Caséines & 26,0 & 21,8 & 142,5 & 152,0 & n.d. $^{1}$ \\
Matières grasses & 0,7 & 6,3 & 2,3 & 39,5 & 390,7 \\
Extraits secs & 89,9 & 85,5 & 225,4 & 268,0 & 443,8 \\
Lactose & 49,4 & 46,4 & 36,6 & 38,0 & 30,7 \\
Cendres & 7,5 & 6,0 & 17,3 & 17,0 & 0,38 \\
Calcium & 1,25 & 0,74 & 5,25 & 4,55 & 0,79 \\
PPL 2 & 0,04 & 1,41 & 0,14 & 8,84 & 0,86 \\
\hline
\end{tabular}

${ }^{1} \mathrm{LUF}=$ rétentat de lait écrémé $\mathrm{UF}, \mathrm{BUF}=$ rétentat de babeurre UF $;$ n.d. $=$ non déterminé. ${ }^{2}$ Phospholipides.

* Ingrédient analysé à chaque production fromagère.

${ }^{1} \mathrm{LUF}=\mathrm{UF}$ skim milk retentate, $\mathrm{BUF}=\mathrm{UF}$ buttermilk retentate; n.d. $=$ non determined. ${ }^{2}$ Phospholipids. * Ingredient analysed during each cheese production. 
Tableau II. Composition $\left(\mathrm{g} \cdot \mathrm{kg}^{-1}\right)$ des laits utilisés pour la fabrication des fromages allégés de type Cheddar.

Table II. Composition $\left(\mathrm{g} \cdot \mathrm{kg}^{-1}\right)$ of cheese milks used to produce low fat Cheddar cheeses.

\begin{tabular}{lrrrrr}
\hline Constituant & LLUF $^{1}$ & LBUF $^{1}$ & LB50 $^{1}$ & BLUF $^{1}$ & ESM $^{2}$ \\
\hline Matières grasses & $20,5^{\mathrm{a}}$ & $20,1^{\mathrm{a}}$ & $20,3^{\mathrm{a}}$ & $19,3^{\mathrm{a}}$ & 0,6 \\
Protéines & $39,9^{\mathrm{a}}$ & $40,1^{\mathrm{a}}$ & $39,8^{\mathrm{a}}$ & $38,8^{\mathrm{b}}$ & 0,2 \\
Caséines & $32,0^{\mathrm{a}}$ & $32,3^{\mathrm{a}}$ & $32,1^{\mathrm{a}}$ & $32,0^{\mathrm{a}}$ & 0,6 \\
$\mathrm{NNC}^{3}$ & $7,9^{\mathrm{a}}$ & $7,8^{\mathrm{a}}$ & $7,7^{\mathrm{a}}$ & $6,8^{\mathrm{a}}$ & 0,5 \\
Extrait sec & $116,2^{\mathrm{a}}$ & $115,9^{\mathrm{ab}}$ & $114,3^{\mathrm{b}}$ & $111,1^{\mathrm{c}}$ & 0,6 \\
Cendres & $8,0^{\mathrm{a}}$ & $8,0^{\mathrm{a}}$ & $8,0^{\mathrm{a}}$ & $8,4^{\mathrm{a}}$ & 0,4 \\
Lactose & $47,9^{\mathrm{a}}$ & $47,8^{\mathrm{a}}$ & $46,3^{\mathrm{b}}$ & $44,2^{\mathrm{c}}$ & 0,4 \\
Calcium & $1,47^{\mathrm{a}}$ & $1,42^{\mathrm{b}}$ & $1,29^{\mathrm{c}}$ & $1,17^{\mathrm{d}}$ & 0,01 \\
Phospholipides & $0,3^{\mathrm{c}}$ & $0,8^{\mathrm{b}}$ & $1,0^{\mathrm{ab}}$ & $1,3^{\mathrm{a}}$ & 0,09 \\
\hline
\end{tabular}

${ }^{1}$ LLUF = lait écrémé + rétentat de lait UF, LBUF = lait écrémé + rétentat de babeurre UF, LB50 = lait écrémé : babeurre (50:50) + rétentat de lait UF : rétentat de babeurre UF (50:50), BLUF = babeurre + rétentat de lait UF.

${ }^{2}$ Erreur standard sur la moyenne. ${ }^{3}$ Azote non caséique $\times 6,38$. ${ }^{\mathrm{a}-\mathrm{d}}$ Les moyennes suivies de lettres différentes dans une même rangée diffèrent à $P \leq 0,05$.

${ }^{1}$ LLUF $=$ skim milk + UF milk retentate, LBUF $=$ skim milk + UF buttermilk retentate, LB50 $=$ skim milk: but termilk (50:50) + UF milk retentate: UF buttermilk retentate (50:50), BLUF = buttermilk + UF milk retentate. ${ }^{2}$ standard error of the means. ${ }^{3}$ Non casein nitrogen $\times 6.38$. ${ }^{\text {a-d }}$ Means with different superscripts in the same row differ at $P \leq 0.05$.

ment plus faibles $(P<0,05)$ pour les laits LB50 et BLUF. Or, ces laits de fromagerie ont été préparés avec une quantité plus importante de babeurre, surtout pour le lait BLUF, dont les teneurs en lactose, en calcium et globalement en protéines étaient plus faibles que celles du lait écrémé (Tab. I). Ces laits (LB50 et BLUF) contenaient également moins de calcium que les autres laits de fromagerie, ils ont donc été supplémentés en calcium tel que décrit précédemment.

Les quatre laits de fromagerie préparés avaient, pour des valeurs en protéines et en matières grasses similaires des teneurs en phospholipides significativement différentes. La concentration en phospholipides des laits LLUF, LBUF, LB50 et BLUF était respectivement de 0,$3 ; 0,8 ; 1,0$ et $1,2 \mathrm{~g} \cdot \mathrm{kg}^{-1}$, ce qui représentait 1,$4 ; 4,3 ; 4,9$ et $6,2 \%$ de phospholipides par rapport aux lipides totaux.

\subsection{Composition des fromages allégés}

La composition ainsi que les valeurs de $\mathrm{pH}$ des fromages allégés sont présentées dans le tableau III. Le pH des fromages était statistiquement semblable pour tous les fromages. La teneur en phospholipides des fromages LLUF, LBUF, LB50 et BLUF était, respectivement, de 1,$3 ; 2,6 ; 5,0$ et $5,6 \mathrm{~g} \cdot \mathrm{kg}^{-1}$ ce qui représentait 0,$7 ; 1,6 ; 3,6$ et $4,4 \%$ de phospholipides par rapport aux lipides totaux. Il est à noter, qu'il n'y a pas de différence significative entre la teneur en phospholipides des fromages due à l'erreur standard sur la moyenne très élevée. 
Tableau III. Composition $\left(\mathrm{g} \cdot \mathrm{kg}^{-1}\right)$ des fromages allégés de type Cheddar.

Table III. Low fat Cheddar cheese composition $\left(\mathrm{g} \cdot \mathrm{kg}^{-1}\right)$.

\begin{tabular}{lccccc}
\hline Constituant & LLUF $^{1}$ & LBUF $^{1}$ & LB50 $^{1}$ & BLUF $^{1}$ & ESM $^{2}$ \\
\hline Humidité & $420,4^{\mathrm{d}}$ & $451,6^{\mathrm{c}}$ & $482,9^{\mathrm{b}}$ & $518,7^{\mathrm{a}}$ & 7,2 \\
$\quad$ HFD $^{3}$ & $51,6^{\mathrm{d}}$ & $53,66^{\mathrm{c}}$ & $56,19^{\mathrm{b}}$ & $59,38^{\mathrm{a}}$ & 0,66 \\
Matières grasses & $185,7^{\mathrm{a}}$ & $158,5^{\mathrm{b}}$ & $140,7^{\mathrm{c}}$ & $126,5^{\mathrm{c}}$ & 5,1 \\
$\quad \mathrm{G} / \mathrm{S}^{3}$ & $32,02^{\mathrm{a}}$ & $28,89^{\mathrm{b}}$ & $27,19^{\mathrm{bc}}$ & $26,27^{\mathrm{c}}$ & 0,73 \\
Protéines & $329,4^{\mathrm{a}}$ & $317,5^{\mathrm{a}}$ & $294,8^{\mathrm{b}}$ & $272,3^{\mathrm{c}}$ & 5,4 \\
Sel & $17,5^{\mathrm{c}}$ & $22,1^{\mathrm{b}}$ & $25,8^{\mathrm{a}}$ & $25,0^{\mathrm{a}}$ & 0,9 \\
$\mathrm{~S}^{\mathrm{a}} \mathrm{H}^{3}$ & $4,15^{\mathrm{b}}$ & $4,89^{\mathrm{a}}$ & $5,32^{\mathrm{a}}$ & $4,82^{\mathrm{a}}$ & 0,19 \\
Cendres & $45,2^{\mathrm{a}}$ & $47,5^{\mathrm{a}}$ & $48,9^{\mathrm{a}}$ & $47,1^{\mathrm{a}}$ & 0,9 \\
$\mathrm{~K}^{3}$ & $0,95^{\mathrm{b}}$ & $1,09^{\mathrm{ab}}$ & $1,15^{\mathrm{a}}$ & $1,13^{\mathrm{a}}$ & 0,05 \\
$\mathrm{Mg}^{3}$ & $0,21^{\mathrm{a}}$ & $0,20^{\mathrm{ab}}$ & $0,18^{\mathrm{bc}}$ & $0,17^{\mathrm{c}}$ & 0,007 \\
$\mathrm{Ca}^{3}$ & $8,64^{\mathrm{a}}$ & $7,93^{\mathrm{b}}$ & $7,11^{\mathrm{c}}$ & $7,08^{\mathrm{c}}$ & 0,20 \\
$\mathrm{P}^{3}$ & $6,21^{\mathrm{a}}$ & $5,60^{\mathrm{b}}$ & $5,46^{\mathrm{bc}}$ & $5,19^{\mathrm{c}}$ & 0,12 \\
$\mathrm{Phospholipides}$ & $1,3^{\mathrm{a}}$ & $2,6^{\mathrm{a}}$ & $5,0^{\mathrm{a}}$ & $5,6^{\mathrm{a}}$ & 1,3 \\
$\mathrm{pH}$ & $5,05^{\mathrm{a}}$ & $5,09^{\mathrm{a}}$ & $5,09^{\mathrm{a}}$ & $5,06^{\mathrm{a}}$ & 0,02 \\
\hline
\end{tabular}

${ }^{1}$ Abréviations voir tableau II. ${ }^{2}$ Erreur standard sur la moyenne. ${ }^{3} \mathrm{HFD}=$ humidité dans la fraction dégraissée $(\%), \mathrm{G} / \mathrm{S}=$ gras sur $\sec (\%), \mathrm{S} / \mathrm{H}=$ sel sur humidité $(\%), \mathrm{K}=$ potassium, $\mathrm{Mg}=$ magnésium, $\mathrm{Ca}=$ calcium, $\mathrm{P}=$ phosphore. ${ }^{\mathrm{a}-\mathrm{c}}$ Les moyennes suivies de lettres différentes dans une même rangée diffèrent à $P \leq 0,05$.

${ }^{1}$ Abbreviations see Table II. ${ }^{2}$ Standard error of the means. ${ }^{3} \mathrm{HFD}=$ moisture in non fat substance $(\%)$, G/S = fat in dry matter $(\%), \mathrm{S} / \mathrm{H}=$ ratio salt on moisture $(\%), \mathrm{K}=$ potassium, $\mathrm{Mg}=$ magnesium, $\mathrm{Ca}=$ calcium, $\mathrm{P}=$ phosphorus. ${ }^{\mathrm{a}-\mathrm{c}}$ Means with different superscripts in the same row differ at $P \leq 0.05$.

La cause probable est la méthode utilisée pour estimer (calcul par différence) la concentration des PPL dans les fromages. L'utilisation de la méthode présentée par Wolff et Castera-Rossignol [36] pour extraire la matière grasse du fromage et doser les PPL aurait probablement été plus précise.

La teneur en humidité et par conséquent l'humidité dans la fraction dégraissée (HFD), ont augmenté avec l'augmentation de la teneur en PPL dans les fromages. C'est le fromage BLUF qui avait significativement la valeur en humidité la plus élevée malgré les ajustements des paramètres de fabrication. De plus, parmi les fromages allégés produits, seul le fromage BLUF n'a pas obtenu la texture caractéristique du Cheddar [25]. Le caillé BLUF était très friable.
Les teneurs en matières grasses et le gras sur sec $(\mathrm{G} / \mathrm{S})$, des fromages ont diminué significativement avec l'augmentation des phospholipides dans le lait de fromagerie. Ce sont les fromages LB50 et BLUF qui avaient les teneurs en matières grasses les plus faibles, en deçà même de la valeur cible en matières grasses de $170 \mathrm{~g} \cdot \mathrm{kg}^{-1}$. Ces résultats indiquent que les caillés LB50 et BLUF n'ont pas été en mesure de bien retenir les matières grasses. Les teneurs en protéines totales ont aussi diminué avec l'augmentation des PPL dans le lait de fromagerie. Cependant, les valeurs protéines sur sec (environ $57 \%$ ) étaient similaires pour tous les fromages, ce qui indique que la rétention des protéines était semblable pour tous les fromages. 
La teneur en sel et le rapport sel sur humidité $(\mathrm{S} / \mathrm{H})$ étaient significativement plus faibles pour le fromage LLUF. Un rapport $\mathrm{S} / \mathrm{H}$ de 5,0 \% était visé pour tous les fromages allégés, ce qui a été impossible d'obtenir pour le fromage LLUF. Il aurait donc été préférable d'ajouter plus de sel au caillé LLUF lors des fabrications. Les teneurs en cendres dans tous les fromages étaient statistiquement similaires quoique légèrement plus faibles pour le fromage LLUF. Par contre, les teneurs en calcium, en magnésium et en phosphore étaient statistiquement plus faibles tandis que les teneurs en potassium étaient plus élevées pour les fromages LB50 et BLUF que pour les autres fromages. Malgré le fait que la teneur en calcium des laits de fromagerie LB50 et BLUF ait été ajustée à $1,4 \mathrm{~g} \cdot \mathrm{kg}^{-1}$, les concentrations en calcium étaient plus faibles dans ces fromages.

\subsection{Composition des lactosérums}

La composition des lactosérums obtenus lors de la fabrication des fromages allégés est présentée dans le tableau IV. La teneur en matières grasses des lactosérums a augmenté avec l'augmentation de la te- neur en PPL dans les laits de fromagerie. Les lactosérums obtenus pour les fromages LB50 et BLUF avaient une teneur en matières grasses significativement plus élevée mais des teneurs en protéines plus faibles. Le lactosérum LBUF avait la teneur en protéines la plus élevée $(P<0,075)$ mais non significativement différente du lactosérum LLUF.

Les teneurs en extraits secs de tous les lactosérums n'étaient pas statistiquement différentes. Par contre, les lactosérums LBUF, LB50 et BLUF avaient une teneur en cendres statistiquement plus élevée que le lactosérum LLUF probablement due à une perte plus importante en calcium, en magnésium et en phosphore (données non mesurées) tel que démontré par la composition des fromages (Tab. III).

La teneur en phospholipides dans les lactosérums était significativement différente. Elle était de 0,$2 ; 0,4 ; 0,5$ et $0,8 \mathrm{~g} \cdot \mathrm{kg}^{-1}$ respectivement pour les lactosérums LLUF, LBUF, LB50 et BLUF, ce qui représentait 6,$6 ; 9,2 ; 9,5$ et $12,6 \%$ de phospholipides par rapport aux lipides totaux. Il est à noter que ces rapports sont plus importants que ceux obtenus pour les laits de fromagerie (1,3 à 6,2\%).

Tableau IV. Composition $\left(\mathrm{g} \cdot \mathrm{kg}^{-1}\right)$ et poids $(\mathrm{kg})$ des lactosérums obtenus lors des fabrications.

Table IV. Composition $\left(\mathrm{g} \cdot \mathrm{kg}^{-1}\right)$ and weight $(\mathrm{kg})$ of whey obtained during cheese making.

\begin{tabular}{lccccc}
\hline Constituant & LLUF $^{1}$ & LBUF $^{1}$ & LB50 $^{1}$ & BLUF $^{1}$ & ESM $^{2}$ \\
\hline Matières grasses & $2,9^{\mathrm{c}}$ & $4,8^{\mathrm{b}}$ & $5,5^{\mathrm{ab}}$ & $6,1^{\mathrm{a}}$ & 0,3 \\
Protéines & $8,9^{\mathrm{ab}}$ & $9,6^{\mathrm{a}}$ & $8,8^{\mathrm{b}}$ & $8,5^{\mathrm{b}}$ & 0,3 \\
Extrait sec & $68,4^{\mathrm{a}}$ & $71,4^{\mathrm{a}}$ & $68,9^{\mathrm{a}}$ & $67,8^{\mathrm{a}}$ & 1,1 \\
Cendres & $6,5^{\mathrm{b}}$ & $6,8^{\mathrm{ab}}$ & $6,9^{\mathrm{ab}}$ & $7,3^{\mathrm{a}}$ & 0,2 \\
Phospholipides & $0,2^{\mathrm{c}}$ & $0,4^{\mathrm{b}}$ & $0,5^{\mathrm{ab}}$ & $0,8^{\mathrm{a}}$ & 0,07 \\
Quantités & $16,65^{\mathrm{a}}$ & $16,66^{\mathrm{a}}$ & $16,55^{\mathrm{b}}$ & $16,48^{\mathrm{b}}$ & 0,03 \\
\hline
\end{tabular}

${ }^{1}$ Abréviations voir tableau II. ${ }^{2}$ Erreur standard sur la moyenne. ${ }^{\text {a-d }}$ Les moyennes suivies de lettres différentes dans une même rangée diffèrent à $P \leq 0,05$.

${ }^{1}$ Abbreviations see Table II. ${ }^{2}$ Standard error of the means. ${ }^{\text {a-d }}$ Means with different superscripts in the same row differ at $P \leq 0.05$. 
Les poids de lactosérum obtenus lors des fabrications fromagères sont également présentés dans le tableau IV. La quantité de lactosérum diminuait avec l'augmentation des PPL dans les laits de fromagerie. Plus la teneur en eau des fromages était élevée, moins la quantité de lactosérum retirée au soutirage était importante. La présence de PPL dans le caillé semble donc permettre une meilleure rétention de l'eau.

\subsection{Rendements fromagers et taux de récupération}

Les rendements fromagers bruts et corrigés calculés pour tous les fromages sont présentés dans le tableau V. Les rendements fromagers bruts ont augmenté significativement avec l'augmentation de la teneur des PPL dans les laits de fromagerie. Ce sont les fromages LB50 et BLUF qui ont obtenu les meilleurs rendements fromagers bruts, soit, respectivement 10,76 et $11,17 \mathrm{~kg} \cdot 100 \mathrm{~kg}^{-1}$. Cependant, les augmentations significatives des rendements bruts ne seraient en fait attribuées qu'à la teneur en eau élevée des fromages; eau apportée par les PPL, puisqu' une fois les rendements ajustés à une teneur constante en eau de $470 \mathrm{~g} \cdot \mathrm{kg}^{-1}$, ils sont statistiquement semblables, voire même légèrement inférieurs pour le fromage BLUF.

Les taux de récupération des protéines $\left(\mathrm{K}_{\mathrm{P}}\right)$ et des matières grasses $\left(\mathrm{K}_{\mathrm{G}}\right)$ sont présentés également dans le tableau V. Le $\mathrm{K}_{\mathrm{P}}$ était statistiquement semblable pour tous les fromages et se situait près de $77 \%$. Ces résultats sont comparables à ceux obtenus par Mistry et al. [27]. Par contre, le $\mathrm{K}_{\mathrm{G}}$ diminuait significativement avec l'augmentation de la concentration en PPL dans les laits de fromagerie. Le fromage fabriqué avec le lait BLUF avait le plus faible $\mathrm{K}_{\mathrm{G}}$ (Tab. V) d'où des teneurs en matières grasses élevées dans le lactosérum BLUF (Tab. IV).

\section{DISCUSSION}

L'ajout combiné de babeurre, de crème, de rétentats de lait UF et de babeurre UF à du lait écrémé a permis de produire des laits de fromagerie dont la teneur en PPL variait de 0,3 à $1,2 \mathrm{~g} \cdot \mathrm{kg}^{-1}$ mais tout en maintenant constantes les teneurs en protéines totales

Tableau V. Rendements fromagers $\left(\mathrm{kg} \cdot 100 \mathrm{~kg}^{-1}\right)$ et taux de récupération (\%) des protéines et des matières grasses des fromages allégés de type Cheddar.

Table V. Cheese yields $\left(\mathrm{kg} \cdot 100 \mathrm{~kg}^{-1}\right)$, fat and protein recoveries (\%) of low fat Cheddar cheese.

\begin{tabular}{lccccc}
\hline & LLUF $^{1}$ & LBUF $^{1}$ & LB50 $^{1}$ & BLUF $^{1}$ & ESM $^{2}$ \\
\hline $\mathrm{RD}_{\mathrm{B}}{ }^{3}$ & $9,44^{\mathrm{c}}$ & $10,04^{\mathrm{b}}$ & $10,76^{\mathrm{a}}$ & $11,17^{\mathrm{a}}$ & 0,16 \\
$\mathrm{RD}_{\mathrm{A}}{ }^{3}$ & $10,45^{\mathrm{a}}$ & $10,40^{\mathrm{a}}$ & $10,38^{\mathrm{a}}$ & $10,04^{\mathrm{a}}$ & 0,16 \\
$\mathrm{~K}_{\mathrm{P}}{ }^{3}$ & $77,16^{\mathrm{a}}$ & $77,74^{\mathrm{a}}$ & $77,47^{\mathrm{a}}$ & $76,23^{\mathrm{a}}$ & 0,99 \\
$\mathrm{~K}_{\mathrm{G}}{ }^{3}$ & $84,65^{\mathrm{a}}$ & $77,28^{\mathrm{b}}$ & $72,66^{\mathrm{bc}}$ & $70,29^{\mathrm{c}}$ & 2,02 \\
\hline
\end{tabular}

${ }^{1}$ Abréviations voir tableau II. ${ }^{2}$ Erreur standard sur la moyenne. ${ }^{3} \mathrm{RD}_{\mathrm{B}}=$ rendement fromager brut, $\mathrm{RD}_{\mathrm{A}}=$ rendement fromager ajusté à $470 \mathrm{~g} \cdot \mathrm{kg}^{-1}$ d'humidité et $22,5 \mathrm{~g} \cdot \mathrm{k} \mathrm{g}^{-1}$ de sel, $\mathrm{K}_{\mathrm{P}}=$ taux de récupération des protéines, $\mathrm{K}_{\mathrm{G}}=$ taux de récupération des matières grasses. ${ }^{\mathrm{a}-\mathrm{c}}$ Les moyennes suivies de lettres différentes dans une même rangée diffèrent à $P \leq 0,05$.

${ }^{1}$ Abbreviations see Table II. ${ }^{2}$ Standard error of the means. ${ }^{3} \mathrm{RD}_{\mathrm{B}}=$ actual cheese yield, $\mathrm{RD}_{\mathrm{A}}=$ cheese yield ad justed to $470 \mathrm{~g} \cdot \mathrm{kg}^{-1}$ of moisture and $22.5 \mathrm{~g} \cdot \mathrm{kg}^{-1}$ of salt, $\mathrm{K}_{\mathrm{P}}=$ protein recovery, $\mathrm{K}_{\mathrm{G}}=$ fat recovery. ${ }^{\mathrm{a}-\mathrm{c}}$ Means with different superscripts in the same row differ at $P \leq 0.05$. 
$\left(40 \mathrm{~g} \cdot \mathrm{kg}^{-1}\right)$ et en matières grasses $\left(20 \mathrm{~g} \cdot \mathrm{kg}^{-1}\right)$. Ces différents laits ont permis de produire des fromages allégés de type Cheddar.

L'ajout de babeurre a affecté la composition des fromages allégés. Plus la teneur en PPL du lait de fromagerie était élevée, plus le fromage allégé était riche en PPL. Une étude réalisée par Sachdeva et Buchheim [31], a démontré que la coagulation du babeurre par de la présure permettait la récupération de $20 \%$ des phospholipides dans le caillé. Selon ces mêmes auteurs, l'augmentation de la teneur en calcium du babeurre diminue les pertes de phospholipides dans le lactosérum. Or, dans la présente étude, les pertes de phospholipides dans les lactosérums étaient en moyenne de $56 \pm$ $9,95 \%$. Ainsi, il a été possible d'obtenir un taux de récupération de phospholipides de $44 \%$ dans les fromages en supplémentant les laits LB50 et BLUF en calcium, en babeurre et en rétentat UF.

Les fromages allégés riches en PPL avaient aussi des teneurs en humidité très élevées. L'ajout de PPL dans le lait de fromagerie a donc nécessité des modifications du procédé de fabrication afin de tenter de diminuer la teneur en eau des fromages. Ainsi, lors de l'étape de coagulation, certains caillés obtenus à partir de laits de fromagerie riches en PPL (LB50 et BLUF) ont été coupés moins fermes [20]. De plus, comme à l'étape du soutirage, l'expulsion de l'eau du caillé est plus efficace à des valeurs plus basses de $\mathrm{pH}$ [24], les lactosérums ont donc été soutirés à des valeurs de $\mathrm{pH}$ plus acide. Malgré les ajustements effectués, il a été impossible d'obtenir des fromages à humidité comparable. Il aurait probablement fallu faire d'autres modifications comme par exemple modifier la température à l'étape de cuisson et du salage, modifier le diamètre des grains du caillé, etc. [9].

D'autres auteurs ont aussi démontré la plus grande rétention d'eau des PPL dans un caillé. Drake et al. [16] ont démontré que des fromages fabriqués avec de la lécithine de soja (phospholipides d'origine végétale) avaient une teneur en humidité plus élevée que le fromage témoin. Il a été démontré également que l'incorporation au lait de fromagerie de PPL sous forme de liposomes (vésicules entourées de plusieurs membranes de PPL et utilisées pour encapsuler des enzymes), provoquait une augmentation de la teneur en eau de fromages standards de type Cheddar, y compris dans le cas où aucune substance n'était encapsulée dans les liposomes [23]. Les résultats de cette étude semblent donc confirmer que les phospholipides possèdent la capacité à retenir l'eau dans le caillé, d'où cette augmentation d'humidité dans les fromages allégés.

Les PPL du babeurre pourraient ne pas être les seuls composés responsables de l'augmentation de la teneur en humidité des fromages. Joshi et al. [21] relient la teneur en humidité plus élevée des fromages contenant du babeurre à la présence de lipoprotéines provenant de la membrane des globules gras du lait. D'autre part, le babeurre provenait d'une crème pasteurisée à haute température $\left(85^{\circ} \mathrm{C}, 18 \mathrm{~s}\right)$ utilisée pour la fabrication du beurre. Lorsque le babeurre est utilisé pour la fabrication fromagère, il doit habituellement subir une autre pasteurisation [21]. Il est connu qu'un traitement thermique entraîne la formation d'interactions entre les protéines sériques, surtout la $\beta$-lactoglobuline et les protéines de la membrane des globules gras [12]. Cette $\beta$-lactoglobuline dénaturée par la chaleur et attachée à la membrane des globules gras se retrouve dans le babeurre après le barattage [15]. Il est aussi connu que l'ajout de protéines sériques dénaturées favorise la rétention d'eau dans le fromage $[7,8,26]$. Joshi et al. [21] stipulent que la deuxième pasteurisation que subit le babeurre a un effet direct sur le procédé de fabrication ainsi que sur la qualité du fromage obtenu. D'après leurs résultats, les caillés contenant du babeurre étaient plus mous, plus friables et avaient une teneur en humidité plus élevée que les fromages témoins. Les résultats de cette étude confirment donc 
leurs observations. De plus, l'incorporation de rétentat LUF ou BUF dans le lait de fromagerie contribue aussi à augmenter la teneur en protéines sériques. Ces dernières une fois dénaturées lors du traitement thermique de pasteurisation $[17,30]$ forment des interactions avec les caséines ce qui affecte la synérèse du caillé [35] et favorise la rétention d'eau dans le fromage $[1,7,8,17$, 26]. Une majoration de $21 \%$ de la teneur en humidité des caillés a pu être observée dans cette étude malgré les modifications apportées lors des fabrications fromagères, comparativement à des augmentations obtenues dans la littérature de 1 à $6 \%$ avec des protéines sériques dénaturées $[7,8,26]$. Toute proportion gardée, les PPL sembleraient permettre une meilleure rétention d'eau dans le caillé que les protéines sériques dénaturées.

L'augmentation de la teneur en humidité a permis d'obtenir des rendements fromagers bruts très élevés. Ces résultats confirment ceux obtenus par Mistry et al. [27], Drake et al. [16] et Hicks et al. [19] qui ont eux aussi obtenu une augmentation du rendement fromager brut lorsque du babeurre UF ou de la lécithine était ajouté au lait de fromagerie. Cette hausse serait aussi partiellement due à l'augmentation de protéines sériques dans le fromage [16]. Cependant, dans cette étude, les rendements bruts obtenus pour les fromages allégés étaient plus élevés d'environ 6,0 à 20,0\% que ceux obtenus par Drake et al. [16] et Mistry et al. [27] en raison surtout du fait que les teneurs en protéines et en matières grasses ont été ajustées respectivement à 40 et $20 \mathrm{~g} \cdot \mathrm{kg}^{-1}$ dans tous les laits de fromagerie.

Les fromages allégées surtout ceux riches en PPL (LB50 et LBUF) en plus d'être très humides contenaient moins de calcium, de magnésium, de phosphore et surtout moins de matières grasses. Les pertes en matières grasses dans les lactosérums étaient très élevées. Mistry et al. [27] ont eux aussi observé que l'ajout de babeurre entraînait des pertes de matières grasses dans le lactosérum. La fermeté du caillé au moment de l'étape de la coagulation peut avoir un impact important sur les pertes dans le lactosérum. Ainsi, le coupage d'un caillé trop mou peut entraîner des pertes de matières grasses et de protéines dans le lactosérum [20]. Or, comparativement aux autres fromages, le caillé BLUF a été coupé après un temps de coagulation de $29 \mathrm{~min}$. De plus, la durée de brassage du caillé dans le lactosérum a été plus longue pour les caillés LB50 et BLUF. L'acidification a donc été plus grande ( $\mathrm{pH}$ de soutirage plus bas), ce qui a entraîné une déminéralisation plus importante des fromages. Une déminéralisation du caillé modifie la microstructure du fromage [6] et réduit la capacité du caillé à retenir les matières grasses [33]. De plus, la teneur en PPL élevée retrouvée dans le fromage BLUF a semblé aussi affecter la formation de la texture du caillé. Ainsi la texture fibreuse typique d'un fromage Cheddar standard ou allégé qui se développe normalement lors de l'étape de cheddarisation [25] n'a pas pu être obtenue pour le fromage BLUF. Le caillé BLUF était très friable. Joshi et al. [21] ont aussi observé que le caillé obtenu à partir d'un lait contenant du babeurre était plus mou et que la texture caractéristique du Cheddar était absente. Cet effet était beaucoup plus marqué lorsque que l'incorporation de babeurre était élevée comme pour le fromage allégé BLUF produit à partir du lait de fromagerie contenant $1,2 \mathrm{~g} \cdot \mathrm{kg}^{-1}$ de PPL. La déminéralisation plus importante du caillé BLUF pourrait aussi en partie expliquer la texture friable obtenue avec ce caillé lors des fabrications.

\section{CONCLUSION}

En combinant du lait, du babeurre et des rétentats de lait et de babeurre UF, il a été possible de faire varier la teneur en PPL dans des laits de fromagerie tout en maintenant constantes les teneurs en protéines et 
en matières grasses. Ces laits de fromagerie ont été utilisés pour fabriquer des fromages allégés de type Cheddar. La composition des fromages allégés était différente selon la teneur en PPL du lait de fromagerie. Ainsi, les laits de fromagerie riches en PPL ont permis de produire des fromages allégés avec des teneurs en humidité élevées, malgré les modifications apportées lors de la fabrication fromagère. Par contre, les teneurs en protéines et en matières grasses de ces mêmes fromages étaient plus basses. Ces résultats confirment donc que le babeurre est un ingrédient de choix pour augmenter facilement la teneur en humidité d'un fromage allégé de type Cheddar. Cependant, cette augmentation semble se faire au détriment d'une perte plus importante des matières grasses dans le lactosérum. Par conséquent, l' augmentation des rendements fromagers observée dans cette étude n'était due qu'à la teneur élevée en eau des fromages et non pas à une meilleure rétention des autres constituants du lait. La concentration maximale en PPL dans un lait de fromagerie standardisé en protéines à $40 \mathrm{~g} \cdot \mathrm{kg}^{-1}$ ne devrait pas dépasser $1,0 \mathrm{~g} \cdot \mathrm{kg}^{-1}$, puisqu'au-delà de cette concentration la formation du caillé lors de la fabrication fromagère est déficiente (texture friable). Ces résultats semblent indiquer que la microstructure du fromage est directement affectée par la présence des PPL dans le lait de fromagerie. Les effets des PPL et des autres constituants du babeurre sur les qualités organoleptiques, l'évolution de la protéolyse, de la texture et de la flore microbienne durant la maturation fromagère des fromages allégés de type Cheddar doivent encore être déterminés.

\section{REMERCIEMENTS}

Cette étude a été supportée par le Conseil de Recherche en Pêche et Agroalimentaire du Québec. Les auteurs tiennent à remercier G. Bélanger, maître fromager au Centre de re- cherche et de développement sur les aliments, pour son aide lors des fabrications fromagères.

\section{RÉFÉRENCES}

[1] Abrahamsen R.K., Cheesemaking from milk fortified with ultrafiltrated whey protein concentrate, Milchwissenchaft 34 (1979) 65-68.

[2] Alais C., Sciences du lait, 4e édition, Édition SEPAIC, Paris, 1984.

[3] Ames B.N., Assay of inorganic phosphate, total phosphate and phosphatase, in: Neufeld E.F., Ginsburg V. (Eds.) Methods in Enzymology, Vol. 8: Complex Carbohydrates, Academic Press, New-York, USA, 1966, pp. 115-117.

[4] AOAC Official Methods of analysis, Vol. II, Chapter 33, 16th edition, Association of Official Analytical Chemist International, 1995.

[5] Atherthon H.V., Newlander J.A., Test for fat: Babcock, Gerber et Mojonnier, in: AVI Publishing Co. (Ed.), Chemistry and Testing of Dairy Products, Wesport, Connecticut, USA, 1977, pp. 71-116.

[6] Audet P., Montpetit D., St-Gelais D., Passey C.A., Effect of protein concentration and milkfat globule size on the development of the microstructure of Cheddar cheese. in: Jouffrey B., Colliex C. (Eds.), Electron Microscopy 1994. Application in Biological Sciences, Vol. 3A, Les Éditions de Physique, Paris, France, 1994.

[7] Baldwin K.A., Baer R.J., Parsons J.G., Seas S.W., Spurgeon K.R., Torrey G.S., Evaluation of yield and quality of Cheddar cheese manufactured from milk with added whey protein concentrate, J. Dairy Sci. 69 (1986) 2543-2550.

[8] Banks J.M., Muir D.D., Effect of incorporation of denatured whey protein on yield and quality of Cheddar cheese, J. Soc. Dairy Technol. 38(1985) 27-32.

[9] Banks J.M., Brechany E.Y., Christie W.W., The production of lowfat Cheddar-type cheese, J. Soc. Dairy Technol. 42 (1989) 6-9.

[10] Bryant A., Ustunol Z., Steffe J., Texture of Cheddar cheese as influenced by fat reduction, J. Food Sci. 60 (1995) 1216-1219.

[11] Christie W.W., Noble R.C., Davies G., Phospholipids in milk and dairy products, J. Soc. Dairy Technol. 42 (1987) 6-9.

[12] Corredig M., Dalgleish D.G., Isolates from industrial buttermilk: Emulsifying properties of materials derived from the milk fat globule membrane, J. Agric. Food Chem. 45 (1997) 4595-4600.

[13] Corredig M., Dalgleish D.G., Buttermilk properties in emulsions with soybean oil as affected by fat globule membrane-derived proteins, J. Food Sci. 63 (1998) 476-480. 
[14] Deeth H.C., The role of phospholipids in the stability of milk fat globules, Aust. J. Dairy Technol. 52 (1997) 44-45.

[15] Drake M.A. Swanson B.G., Reduced and low fat cheese technology: A review, Trends Food Sci. Technol. 6 (1995) 366-369.

[16] Drake M.A., Chen X.Q., Gerard P.D., Gurkin S.U., Composition and quality attributes of reduced-fat cheese as affected by lecithine type, J. Food Sci. 63 (1998) 1018-1023.

[17] Guinee T.P., Fenelon M.A., Mulholland E.O., O'Kennedy B.T., O'Brien N., Reville W.J., The influence of milk pasteurization temperature and $\mathrm{pH}$ at curd milling on the composition, texture and maturation of reduced fat Cheddar cheese, Int. J. Dairy Technol. 51 (1998) 1-10.

[18] Hardy J., L'activité de l'eau et le salage des fromages, in: Eck A., Gillis J.-C. (Eds.), Le Fromage, Édition Lavoisier, Technique et Documentation, Paris, 1997 pp. 62-85.

[19] Hicks C.L., O'Learly J., Holbrook J., Effect of lecithin on cheese yield, J. Dairy Sci. 68 (1985) 1603-1607.

[20] Hill A.R., Chemical species in cheese and their origin in milk components, in Chemistry of Structure-Function Relationships in: Malin E.L., Tunick M.H.(Eds.), Cheese, Advances in Experimental Medecine and Biology, Vol. 367, Plenum Press, New-York, USA, 1995 pp. 43-58.

[21] Joshi N.S., Thakar P.N., Jana A.H., Utilization of butter milk in cheese making-A review, Indian Food Packer, March-April (1994) 59-65.

[22] Kosikowski F.V., Mistry V.V., Cheddar cheese and related types, in: Kosikowski F.V., Mistry V.V. (Eds.), Cheese and fermented milk foods, Vol. 1: Origins and Principles, Westport, Connecticut, USA, 1997, pp. 204-225.

[23] Laloy E., Vuilllemard J.C., Simard R.E., Characterization of liposomes and their effect on the properties of Cheddar cheese during ripening, Lait 78 (1998) 401-412.

[24] Lawrence R.C., Heap H.A., Gilles J., A controlled approach to cheese technology, J. Dairy Sci. 67 (1984) 1632-1645.

[25] Lawrence R.C., Gilles J., Creamer L.K., Cheddar cheese and related dry-salted cheese varieties, in:
Fox P.F. (Ed.), Cheese: Chemistry, Physics and Microbiology, Vol. 2, Major Cheese Groups, Chapman \& Hall, London, 1993, pp. 1-38.

[26] Lo C.G., Bastian E.D., Incorporation of native and denatured whey proteins into cheese curd for manufacture of reduced fat, Havarti-type cheese, J. Dairy Sci. 81 (1998) 16-24.

[27] Mistry V.V., Metzger L.E., Maubois J.L., Use of ultrafiltered sweet buttermilk in the manufacture of reduced fat Cheddar cheese, J. Dairy Sci. 79 (1996) 1137-1145.

[28] Pierre A., Le Graet Y., Daufin G., Michel F., Gésan G., Whey microfiltration performance: influence of protein concentration by ultrafiltration and of physicochemical pretreatment, Lait 74 (1994) 65-77.

[29] Rodriguez J., Recent advances in the development of low-fat cheeses, Trends Food Sci. Technol. 9 (1998) 249-254.

[30] Rodriguez J., Requena T., Juarez M., Process for low-fat cheese from ultrafiltered milk, J. Food Sci. 63 (1998) 665-667.

[31] Sachdeva S., Buchheim W., Recovery of phospholipids from buttermilk using membrane processing, Kiel. Milchwirt. Forschungsber. 49 (1997) 47-68.

[32] SAS Institute Inc., SAS/STAT ${ }^{\circledR}$ User's Guide, Version 6, 4th edition, Vol. 2, Cary, NC, SAS Institue Inc., 1989.

[33] St-Gelais D., Passey C.A., Haché S., Roy P., Production of low-fat Cheddar Cheese with low and high mineral retentate powders and different fractions of milkfat globules, Int. Dairy J. 7 (1997) 733-741.

[34] Théodet C., Gandemer G., Comparaison de cinq méthodes pour extraire les lipides du lactosérum et de ses dérivés, Lait 71 (1990) 41-54.

[35] Walstra P., van Dijk H.J.M., Geurts T.J., The syneresis of curd. 1- General considerations and literature review, Neth. Milk Dairy J. 39 (1985) 209-246.

[36] Wolff R.L., Castera-Rossignol F.M., Mise au point etévaluation d'une méthode d'extraction de la matière grasse de fromage de type emmental, Rev. Fr. Corps Gras 3 (1987) 123-132.

To access this jounal online: www.edpsciences.org 